\title{
DIRECT FINITENESS OF CERTAIN MONOID ALGEBRAS
}

\author{
by W. D. MUNN
}

(Received 8th September 1994)

\begin{abstract}
A semigroup is said to be completely regular if and only if each of its elements lies in a subgroup. It is shown that the algebra of a completely regular monoid (semigroup with identity) over a field of characteristic zero is directly finite.
\end{abstract}

1991 Mathematics subject classification: 16S36, $20 \mathrm{M} 25$.

A ring $R$ with unity 1 is termed directly finite (or von Neumann finite) if and only if, for all $a, b \in R, a b=1$ implies $b a=1$. Kaplansky [3] has shown that the group algebra of an arbitrary group over a field of characteristic zero is directly finite. The purpose of this note is to generalise Kaplansky's result from group algebras to a wider class of monoid algebras, namely those in which the monoids are completely regular.

To facilitate the discussion, it is convenient to introduce a further concept. A ring $R$ is said to be quasidirectly finite if and only if, for all $a, b \in R, a b=a+b$ implies $a b=b a$. It is easily seen that, for the case in which $R$ has a unity, direct finiteness and quasidirect finiteness are equivalent properties. However, the second property can be useful in the study of a monoid algebra-for, in general, there are important auxiliary semigroup algebras that need not have unity elements. In fact we shall show that the algebra of a completely regular semigroup over a field of characteristic zero is quasidirectly finite. The result stated in the summary above is an immediate consequence.

We begin with an elementary lemma that provides a basis for induction.

Lemma 1. Let $R$ be a ring, let $S$ be a subring of $R$ and let $T$ be an ideal of $R$ such that $R=S \oplus T$. Then $R$ is quasidirectly finite if and only if $S$ and $T$ are quasidirectly finite.

Proof. It is clear that if $R$ is quasidirectly finite then so also are $S$ and $T$. Assume, conversely, that $S$ and $T$ are quasidirectly finite and suppose that $a, b \in R$ are such that $a b=a+b$. Write $a=a_{1}+a_{2}$ and $b=b_{1}+b_{2}$, where $a_{1}, b_{1} \in S$ and $a_{2}, b_{2} \in T$. Then

$$
\begin{gathered}
a_{1} b_{1}=a_{1}+b_{1}, \\
a_{2} b_{2}+a_{1} b_{2}+a_{2} b_{1}=a_{2}+b_{2} .
\end{gathered}
$$


Since $S$ is quasidirectly finite, (1) implies that

$$
b_{1} a_{1}=b_{1}+a_{1} .
$$

Now let $R^{1}$ be an overring of $R$ containing a unity 1 . We operate in $R^{1}$ and deduce results on $R$ itself. From (3), the equation

$$
\left(1-b_{1}\right)\left(1-a_{1}\right)=1
$$

holds in $R^{1}$. Thus, from (4) and (2),

$$
a_{2}\left(1-b_{1}\right)\left(1-a_{1}\right) b_{2}=a_{2}\left(1-b_{1}\right)+\left(1-a_{1}\right) b_{2} .
$$

Hence, since $a_{2}\left(1-b_{1}\right)$ and $\left(1-a_{1}\right) b_{2}$ lie in $T$ and this ring is quasidirectly finite,

$$
\left(1-a_{1}\right) b_{2} a_{2}\left(1-b_{1}\right)=\left(1-a_{1}\right) b_{2}+a_{2}\left(1-b_{1}\right) .
$$

Pre- and post-multiplying both sides of (5) by $1-b_{1}$ and $1-a_{1}$ respectively, and using (4), we see that

$$
b_{2} a_{2}=b_{2}\left(1-a_{1}\right)+\left(1-b_{1}\right) a_{2}
$$

that is,

$$
b_{2} a_{2}+b_{1} a_{2}+b_{2} a_{1}=b_{2}+a_{2}
$$

Finally, (3) and (6) combine to give $b a=b+a$. Thus $R$ is quasidirectly finite.

It is easy to deduce from Lemma 1 (or indeed to show directly) that if $R$ is a quasidirectly finite ring and the ring $R^{1}$ is formed by adjoining a unity to $R$ in the usual way then $R^{1}$ is directly finite.

By a semilattice we mean a commutative semigroup consisting of idempotents. A ring $R$ is said to be graded by a semilattice $Y$ if and only if $R$ has a family of subrings $R_{\alpha}\left(\alpha \in Y\right.$ ) (called the homogeneous components of $R$ ) such that $R=\bigoplus_{\alpha \in Y} R_{\alpha}$ and, for all $\alpha, \beta \in Y, R_{\alpha} R_{\beta} \subseteq R_{\alpha \beta}$. The main result below relies on the fact that the semigroup algebras that we consider have a natural semilattice-grading.

First, we establish

Lemma 2. Let $R$ be a semilattice-graded ring. Then $R$ is quasidirectly finite if and only if each of its homogeneous components is quasidirectly finite.

Proof. Let $R=\bigoplus_{\alpha \in Y} R_{\alpha}$, where $Y$ is a semilattice, each $R_{\alpha}(\alpha \in Y)$ is a subring of $R$ and, for all $\alpha, \beta \in Y, R_{\alpha} R_{\beta} \subseteq R_{\alpha \beta}$. Clearly, if $R$ is quasidirectly finite then so is each $R_{\alpha}$. Now suppose that, for all $\alpha \in Y, R_{\alpha}$ is quasidirectly finite. For $x \in R$, we denote the $R_{\alpha}$-component of $x$ by $x_{\alpha}(\alpha \in Y)$. 
Let $a, b \in R$ be such that $a b=a+b$. We may assume that $a$ and $b$ are both nonzero. Let $Z$ denote the subsemigroup of $Y$ generated by the (finite) subset $\left\{\alpha \in Y: a_{\alpha} \neq 0\right.$ or $\left.b_{\alpha} \neq 0\right\}$. Since $Y$ is locally finite, $Z$ is a finite semilattice. Write $S:=\bigoplus_{\alpha \in Z} R_{\alpha}$. Then $S$ is a subring of $R$ containing $a$ and $b$ and it suffices to show that $S$ is quasidirectly finite. Note first that $Z$ is partially ordered by the rule that

$$
\alpha \leqq \beta \Leftrightarrow \alpha \beta(=\beta \alpha)=\alpha \quad(\alpha, \beta \in Z) .
$$

Let $n=|Z|$ and construct subsets $Z_{1}, Z_{2}, \ldots, Z_{n}$ of $Z$ successively by taking $Z_{1}=\{\omega\}$, where $\omega$ is the least element of $Z$, and $Z_{i+1}=Z_{i} \cup\{\alpha\}$ if $1 \leqq i<n$, where $\alpha$ is minimal in $Z \backslash Z_{i}$ under the partial ordering. Write $T_{i}:=\bigoplus_{\alpha E Z_{i}} R_{\alpha}(i=1,2, \ldots, n)$. It is clear that each $T_{i}$ is an ideal of $S$ and that

$$
T_{1} \subset T_{2} \subset \ldots \subset T_{n}=S
$$

Now $T_{1}$ is quasidirectly finite, since $T_{1}=R_{\omega}$. Assume that $n>1$ and that $T_{i}$ is quasidirectly finite for $i<n$. By definition, $T_{i+1}=R_{\alpha} \oplus T_{i}$ for some $\alpha \in Z$. Hence, by Lemma $1, T_{i+1}$ is quasidirectly finite. Thus, by induction, we see that $S$ is quasidirectly finite, as required.

We adopt the basic terminology and notation for semigroups established in [2] (with the exception of the now-standard phrase 'completely regular' (see below)). Throughout, the symbol $F$ denotes a field. The semigroup algebra $[2, \S 5.2]$ of a semigroup $S$ over $F$ is denoted by $F[S]$ and, for a positive integer $n$, the $F$-algebra consisting of all $n \times n$ matrices over an $F$-algebra $R$ (under the usual operations) is denoted by $M_{n}(R)$.

The following result was obtained by Kaplansky [3, p. 122]. (See also [4] and [5, Corollary 2.1.9 and Example 9, p. 65].)

Lemma 3. Let $F$ have characteristic zero and let $G$ be a group. Then, for all positive integers $n, M_{n}(F[G])$ is directly finite.

From Lemma 3 we derive

Lemma 4. Let $F$ have characteristic zero and let $S$ be a completely simple semigroup. Then $F[S]$ is quasidirectly finite.

Proof. By Rees's theorem [2, Theorem 3.5], $S \cong \mathscr{M}(G ; I, \Lambda ; P)$ for some group $G$, some nonempty sets $I$ and $\Lambda$ and some $\Lambda \times I$ matrix $P$ over $G$. Then, as in [2, Lemma 5.17], without loss of generality we can assume that $F[S]=\mathscr{M}(F[G] ; I, \Lambda ; P)$, the algebra of all $I \times \Lambda$ matrices over $F[G]$ having at most finitely many nonzero entries, with the usual addition and scalar multiplication, and with multiplication $\circ$ defined in terms of ordinary matrix multiplication by

$$
X \circ Y=X P Y \quad(X, Y \in F[S])
$$


Let $A, B \in F[S]$ be such that $A \circ B=A+B$. We have to show that $A \circ B=B \circ A$. Choose nonempty finite subsets $I_{1}$ and $\Lambda_{1}$ of $I$ and $\Lambda$, respectively, such that all nonzero entries of $A$ and $B$ in each case lie in the $I_{1} \times \Lambda_{1}$ submatrix. Let $T$ be the subalgebra of $F[S]$ generated by $A$ and $B$. Then each element of $T$ is such that all entries lying outside the $I_{1} \times \Lambda_{1}$ submatrix are zero. Let $P_{1}$ denote the $\Lambda_{1} \times I_{1}$ submatrix of $P$ and let $M$ denote the algebra $\mathscr{M}\left(F[G] ; I_{1}, \Lambda_{1} ; P_{1}\right)$. Clearly, the mapping from $T$ into $M$ defined by $X \mapsto X_{1}(X \in T)$, where $X_{1}$ is the $I_{1} \times \Lambda_{1}$ submatrix of $X$, is an injective algebra homomorphism. Thus

$$
A_{1} P_{1} B_{1}=A_{1}+B_{1}
$$

and it suffices to show that $A_{1} P_{1} B_{1}=B_{1} P_{1} A_{1}$.

Let $N$ denote the $F$-algebra of all $I_{1} \times I_{1}$ matrices over $F[G]$ under the usual operations. Now $A_{1} P_{1}$ and $B_{1} P_{1}$ lie in $N$; and, from (1), $\left(A_{1} P_{1}\right)\left(B_{1} P_{1}\right)=A_{1} P_{1}+B_{1} P_{1}$. But, by Lemma 3 (since $I_{1}$ is finite), $N$ is directly finite and so quasidirectly finite. Hence $A_{1} P_{1}+B_{1} P_{1}=\left(B_{1} P_{1}\right)\left(A_{1} P_{1}\right)$. Post-multiplying by $B_{1}$ and applying (1), we find that

$$
A_{1} P_{1} B_{1}+B_{1} P_{1} B_{1}=B_{1} P_{1} A_{1} P_{1} B_{1}=B_{1} P_{1}\left(A_{1}+B_{1}\right)=B_{1} P_{1} A_{1}+B_{1} P_{1} B_{1} \text {. }
$$

Thus $A_{1} P_{1} B_{1}=B_{1} P_{1} A_{1}$.

Remark. The same argument yields the more general result that the contracted semigroup algebra $F_{0}[S]$ of a completely 0 -simple semigroup $S$ over a field $F$ of characteristic zero is quasidirectly finite. (Contracted semigroup algebras are defined in $[2, \S 5.2]$.)

A semigroup $S$ is said to be completely regular if and only if each element of $S$ lies in a subgroup of $S$; that is, if and only if $S$ is a union of groups. Such semigroups (under the title 'semigroups admitting relative inverses') were first studied by Clifford [1], who characterised them as semilattices of completely simple semigroups (see [2, Chapter 4]).

We now have the

Theorem. Let $F$ be a field of characteristic zero and let $S$ be a completely regular semigroup. Then $F[S]$ is quasidirectly finite.

Proof. By [2, Theorem 4.6], there exists a semilattice $Y$ and a family of pairwisedisjoint completely simple semigroups $S_{\alpha}(\alpha \in Y)$ such that $S=\bigcup_{\alpha \in Y} S_{\alpha}$ and, for all $\alpha, \beta \in Y, S_{a} S_{\beta} \subseteq S_{\alpha \beta}$. (In fact, $Y$ is isomorphic to the semilattice of principal ideals of $S$ and the $S_{\alpha}$ are the $\mathscr{J}$-classes of $S$.) Then $F[S]=\bigoplus_{\alpha \in Y} F\left[S_{\alpha}\right]$ and, for all $\alpha, \beta \in Y$, $F\left[S_{\alpha}\right] F\left[S_{\beta}\right] \subseteq F\left[S_{\alpha \beta}\right]$; that is, $F[S]$ is graded by $Y$ and has homogeneous components $F\left[S_{\alpha}\right](\alpha \in Y)$. By Lemma 4, each $F\left[S_{\alpha}\right]$ is quasidirectly finite. Hence, by Lemma $2, F[S]$ is quasidirectly finite.

Corollary. Let $F$ be a field of characteristic zero and let $S$ be a completely regular monoid. Then $F[S]$ is directly finite. 
Remark. Since, for any positive integer $n, M_{n}(F[G])$ is directly finite when $G$ is an abelian group and $F$ is an arbitrary field, a theorem analogous to that above can be obtained by replacing the hypothesis that $F$ has characteristic zero by the requirement that every subgroup of $S$ be abelian. In particular, it follows that if $F$ is an arbitrary field and $S$ is a band (that is, a semigroup of idempotents) then $F[S]$ is quasidirectly finite.

\section{REFERENCES}

1. A. H. Clifford, Semigroups admitting relative inverses, Ann. of Math. 42 (1941), 1037-1049.

2. A. H. Clifford and G. B. Preston, The algebraic theory of semigroups, vol. 1 (Math. Surveys 7, Amer. Math. Soc., Providence R.I., 1961).

3. I. KAPLANSKy, Fields and rings (Chicago Lectures in Math., Univ. of Chicago, 1969).

4. M. S. Montgomery, Left and right inverses in group algebras, Bull. Amer. Math. Soc. 75 (1969), 539-540.

5. D. S. Passman, The algebraic theory of group rings (Wiley-Interscience, New York, 1977).

Department of Mathematics

UNIVERSITY OF GLASGOW

GlasGow G12 8QW

SCOTLAND 